\title{
Military Occupation
}

National Cancer Institute

\section{Source}

National Cancer Institute. Military Occupation. NCI Thesaurus. Code C97647.

An employment class consisting of all persons employed in the military. 